\title{
Prevalencia de hábitos, conductas e intereses de estudiantes de medicina de 5 escuelas del país
}

BENJAMÍN HORWITZ $^{(1)}$, RUBÉN MIRANDA ${ }^{(2)}$, ÁLVARO WOLFENSON ${ }^{(2)}$, FELIPE SANTELICES $^{(2)}$, LUIS MARTÍNEZ ${ }^{(3)}$, PALOMA DOMANCIC ${ }^{(1)}$, CAROLINA ROMERO $^{(1)}$ Y MIVIALA BUSTAMANTE $^{(1)}$

\section{RESUMEN}

Los estudiantes de medicina dedican gran parte del tiempo a estudiar las materias necesarias para su preparación profesional. Así, logran un adecuado desarrollo de conocimientos científicos y competencias médicas. Sin embargo, los médicos necesitan desarrollar habilidades humanísticas.

Su objetivo es conocer prevalencias de hábitos, conductas e intereses de alumnos de medicina de distintas escuelas del país, públicas y privadas, en áreas culturales, recreativas, deportivas, actividades comunitarias y de ayuda social, religiosas, políticas, y trabajos remunerados.

Se realizó una encuesta anónima a 299 alumnos de medicina de 5 Universidades del país de primero, tercero, y séptimo año; elegidos al azar, en aspectos antes mencionados. Los datos se analizaron con el programa EpiInfo 2002.

Fuman: 31,11\%. Bebe alcohol: 73,91\%. Leen periódicos: 75,59\%, priorizan noticias nacionales y culturales. Actividades recreativas no deportivas y deportivas más de una vez al mes: $51,2 \%$ y $51,51 \%$. Un 27,09\% realiza actividades comunitarias, considerándolas importante un 79,26\%. Un 61,54\% manifiesta interés por política, sólo un 55,85\% está inscrito en el Servicio Electoral. Un 71,57\% adhiere a alguna religión, un 70\% se declara católico. No trabaja un 77,93\%, la mayoría por no tener tiempo. Los que trabajan, 62,5\% lo hacen para tener dinero "extra".

Casi la mitad de los estudiantes de medicina dedica parte de su tiempo a actividades culturales, y a pesar de que la mayoría considera importante para su formación la práctica de actividades comunitarias, pocos las realizan. En las escuelas de medicina no hay un claro incentivo para el desarrollo de las artes y letras, por lo que creemos debe impulsarse la integración de las humanidades médicas en la malla curricular de la carrera de medicina.

Palabras Claves: Humanidades Médicas, Estudiantes Medicina.

\section{ABSTRACT}

PREVALENCE OF HABITS, BEHAVIORS AND INTERESTS AMONG MEDICAL STUDENTS FROM 5 MEDICAL SCHOOLS IN CHILE.

Most of their time, medical students are engaged in studying the courses needed for their professional training. Thus, they develop an adequate scientific knowledge and acquire medical skills. However, physicians also need to develop humanistic abilities.

The purpose of the present work is to assess the prevalence of habits, behaviors and interests

\footnotetext{
(1) Interno de Medicina. Universidad de Chile. bhorwitz@gmail.com

(2) Interno de Medicina. Universidad de Santiago de Chile.

(3) Facultad de Ciencias Médicas. Departamento de Salud Pública y Epidemiología. Universidad de Santiago de Chile.
} 
among medical students from different public and private medical schools across the country, considering the areas of culture, leisure, sports, community and social care, religion, politics and paid jobs.

An anonymous survey was carried out among 299 randomly selected medical students from 5 Chilean Universities who were in the first, the third and the seventh year of their career. Data analysis was performed with EpiInfo 2002.

The results were as follows: $31,11 \%$ were smokers; $73.91 \%$ drank alcohol; $75.59 \%$ read the newspapers, favoring national and culture news; $51.2 \%$ and $51.51 \%$ performed non-sport leisure and sport activities more than once a month; $27.09 \%$ were involved in community aid, and $79.26 \%$ considered such activities as important; $61.54 \%$ expressed interest in politics, and only $55.85 \%$ were listed on the electoral roll; $71.57 \%$ adhered to any religion, $70 \%$ declared themselves Catholics; $77.93 \%$ did not work, most of them due to lack of time; among those who worked, $62.5 \%$ did it to obtain extra money.

Almost half of medical students spend part of their time in cultural activities, and although most of the students consider community care as an important factor for their training, very few put into practice such activities. Medical schools do not encourage clearly enough the development of arts and letters, therefore the integration of medical humanities to the medical curriculum should be encouraged.

Keywords: medical humanities, medical students

\section{INTRODUCCIÓN}

Los estudiantes de medicina dedican gran parte de su tiempo a estudiar las materias necesarias para su preparación profesional. Con esto, logran un adecuado desarrollo en sus conocimientos científicos y competencias médicas. Sin embargo, necesitan desarrollar habilidades humanísticas. Estas nos permiten considerar a las personas como individuos íntegros, y reconocer aspectos relevantes de su calidad de vida. Estas habilidades comprenden las áreas literarias, filosóficas, musicales, teatrales, artes en la comunidad, artes visuales, entre otras ${ }^{1}$. Es muy importante el rol de la palabra y el lenguaje en el abordaje anamnéstico y en la terapéutica, los cuales se desarrollan a través de cursos de humanidades ${ }^{2,3}$.

La filosofía ha estado presente en la medicina desde sus inicios, y en ésta se incluyen temas éticos y los dilemas a los que se ven enfrentados los médicos en su quehacer diario. El interés por la filosofía nos permite analizar los problemas, considerar los argumentos expuestos en ellos, y aclarar nuestras ideas y pensamientos en cuanto a cómo nos sentimos, cómo sabemos, y cómo podríamos actuar ${ }^{1}$. Asimismo, las humanidades nos permiten comprender la manera de sentir de las otras personas, y ver los problemas desde su perspectiva ${ }^{1}$.

Hasta hace unos 50 años, en la enseñanza mundial del arte médica se esperaba que los médicos en formación adquirieran ciertas habilidades en las artes, filosofía, retórica o lógica. Esto debido a que se sabe que las humanidades facilitan la educación, la terapéutica, y el bienestar de las personas. $\mathrm{La}$ medicina es una ciencia social no exacta que trabaja con personas, por lo que es fundamental el desarrollo de estas habilidades para un buen manejo de la relación médico-paciente. Es por ello que desde hace más de dos décadas el tema ha sido motivo de seminarios, trabajos de investigación, debates; y se han creado centros para el estudio de las artes y las humanidades. La enseñanza de las artes entre los profesionales médicos se ha incrementado sobre todo en el mundo desarrollado, donde las humanidades están integradas en el currículum de las escuelas de medicina, se desarrollan seminarios, cursos 
y discusiones en aumento constante. Las encuestas a quienes han sido sometidos a dichas mallas revelan que los estudiantes las consideran una herramienta muy valiosa para reflexionar acerca de la vida, la calidad de vida, y cuestiones clínicas desde una perspectiva distinta. Es tal el nivel de desarrollo de estos países que los hospitales incorporan el arte a la ornamentación de su infraestructura.

De la experiencia en América Latina uno de los países con mayor desarrollo en algunas de sus escuelas es Argentina. Ellos tienen más 20 años de experiencia en el desarrollo y enseñanza de las humanidades médicas ${ }^{4}$. Desde 1976 se implementan cursos electivos acerca de las humanidades, y en 1980 crean el instituto de humanidades médicas 5 . Ellos fueron los primeros en acuñar el término "kalología médica", del griego "Kalòs" (bonito) que se refiere a la belleza en la música y la danza, y que es ampliamente reconocido como término que reúne el conjunto de disciplinas que involucra la kalología. Asimismo, otro término utilizado es el de "philokalia" (del griego philos: amigo, y kalia: belleza $)^{6}$. Actualmente su nuevo currículum de medicina, que incluye las humanidades médicas, está en evaluación.

Además de su relación con los pacientes, los médicos pueden tener un rol importante en la política de una nación. Históricamente los médicos han sido participantes de la contienda política de los países, a través de senadurías, alcaldías, parlamento, dirigencia de partidos políticos, etc ${ }^{7}$. Y Chile no es la excepción.

Un hecho preocupante es la poca participación de los jóvenes de Chile en lo que respecta a la política y desarrollo de las artes, a pesar de que en el último tiempo se ha visto un incremento en dicha materia. Según una encuesta del INJUV de (1998): los jóvenes leen poco, practican poco deporte y ven mucha televisión. El 83\% ve televisión y escucha radio diariamente, sólo un 30\% lee diarios o revistas, y apenas un $25,6 \%$ practica deportes ${ }^{8}$. Según otra encuesta del INJUV del año 2000, más de un $60 \%$ de los jóvenes de entre 20 y 24 años no está inscrito en el registro electoral ${ }^{9}$. Al año 2005, el total de jóvenes entre 20 a 24 años inscritos en el registro electoral es de 281.153 personas, correspondiente a un $21,3 \%$ de jóvenes inscritos de ese grupo etario. Los estudiantes de medicina pertenecen en su mayoría a este grupo.

\section{OBJETIVO}

Conocer la prevalencia de hábitos, conductas e intereses de los alumnos de medicina de distintas escuelas del país, incluidas públicas y privadas, en áreas culturales, recreativas, deportivas, actividades comunitarias y de ayuda social, actividades religiosas, actividades políticas, y trabajos remunerados.

\section{MATERIAL Y MÉTODO}

Se desarrolló una investigación exploratoria, descriptiva de tipo transversal, mediante la aplicación de una encuesta que contiene 35 preguntas, diseñada por los autores con la revisión por expertos en investigación social y epidemiológica. En ella se consulta acerca de hábitos (consumo de alcohol y tabaco), realización de actividades recreativas no deportivas, práctica de actividades deportivas, participación en tareas de ayuda social y comunitaria, intereses culturales, políticos, y religiosos. Como variable de confusión se consultó acerca del desempeño de trabajos remunerados que dificulten la práctica de las actividades antes mencionadas. El cuestionario se aplicó a estudiantes de medicina de primer, tercer y séptimo año (15 a 20 encuestados por curso, elegidos en forma aleatoria, mediante enumeración según listado oficial del curso y elección por tabla de números aleatorios) de 5 escuelas de medicina del país, las que incluyen: Universidad de Chile, Universidad de Santiago de Chile, Universidad Mayor, Universidad Austral, Universidad de Concepción. La encuesta fue aplicada entre septiembre y diciembre de 2005. Los resultados de las encuestas fueron tabulados con el programa MS Excel 2003; se realizó un análisis de distribución de frecuencias absolutas, y se estableció la prevalencia porcentual de las variables de interés mediante 
el software EpiInfo 2000. Además, se establecen intervalos de confianza (IC) al 95\% para cada prevalencia de hábitos, conductas e intereses más relevantes.

\section{RESULTADOS}

Las características del grupo encuestado se presentan en la Tabla 1.

Con respecto a los hábitos, 99 estudiantes declaran fumar, lo que corresponde a un $31,11 \%$ (95\% IC: $27,8 \%$ - 38,4\%); y de ellos, un 63,27\% fuma entre 1 y 5 cigarrillos al día, un $27,55 \%$ fuma entre 6 y 10 cigarrillos al día, y los restantes fuman más de 10 cigarrillos al día. 70 de los estudiantes que fuman, que corresponden a un $72,16 \%$ (95\% IC: 60,3\% - 80,9\%) refieren fumar más desde que ingresaron a la carrera de medicina. En lo referente al consumo de bebidas alcohólicas, 221 encuestados declaran beberlas (73,91\%, 95\% IC: 68,9\% - 78,9\%); y de ellos, 9 dicen hacerlo diariamente (3,91\%), y 148 $(64,35 \%)$ lo hacen sólo durante los fines de semana. El resto consume alcohol en forma esporádica (una vez al mes o menos). Se destaca que el $27,84 \%$ de los encuestados dice no beber alcohol en lo absoluto.

En cuanto a las actividades recreativas no deportivas, se observó que tan solo un 51,2\% (95\% IC: 45,5\% - 56,9\%) de la muestra dedica parte de su tiempo libre al arte y cultura por lo menos una vez al mes (teatro, ópera, cine, música, museos, etc.). Los detalles sobre estos resultados se muestran en la Figura 1. Un 75,59\% (226 personas, 95\% IC: 70,7\% - 80,5\%) declara leer el diario, y de ellos un $22,07 \%$ lo hace todos los días y un 28,76\% los lee sólo los fines de semana. Asimismo, de los encuestados que leen el diario, un $29,1 \%$ prioriza las noticias nacionales, y un $26,42 \%$ dejan las noticias de farándula como última prioridad. En la Figura 2 se detallan los resultados acerca del tiempo diario que dedican los encuestados a ver televisión; escuchar radio; leer libros, periódicos o revistas; y a navegar y chatear por Internet. Queremos destacar que 160 de ellos (53,5\%, 95\% IC: 47,9\% - 59,2\%) no le dedican tiempo diariamente a leer libros

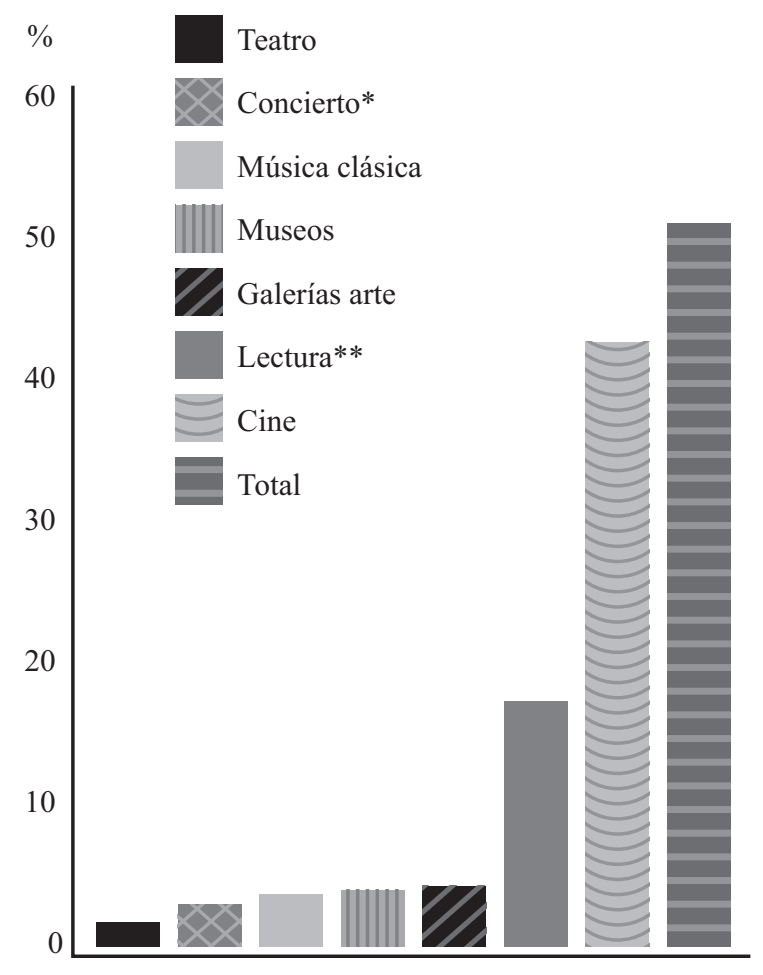

Figura 1. Actividades recreativas no deportivas practicadas por lo menos $1 \mathrm{vez}$ al mes.

* Concierto de cualquier tipo de música, excepto clásica.

** Lectura de libros no relacionados con la carrera de Medicina.

que no estén relacionados con la carrera.

Un 51,51\% de la muestra (154 estudiantes, 95\% IC: 45,9\% - 57,2\%) refiere practicar deportes; y de ellos, 143 (47,83\% del total de la muestra) lo hace por lo menos una vez a la semana. Del total de los encuestados, sólo un 27,09\% (95\% IC: 22,1\% - 32,1\%) declara participar actualmente en actividades comunitarias (de ayuda social), y de ellos el $87,6 \%$ comenzó a hacerlo antes de entrar a la carrera o durante el primer año. Del total de los encuestados, 237 personas $(79,26 \%)$ consideran importante que los estudiantes de medicina realicen estas actividades para su formación profesional.

De los 299 participantes en el estudio, 214 (71,57\%, 95\% IC: 66,5\% - 76,7\%) declaran tener alguna religión. De esos estudiantes, un 
$86,45 \%$ adhiere a la misma religión que practican sus padres. Sin embargo, sólo un $55,14 \%$ se declara practicante, y un $43,93 \%$ realiza actividades relacionadas a ella. Las religiones que practican los estudiantes de medicina se detallan en la Figura 3. Se destaca que un 70\% (95\% IC: $63,9 \%-76,1 \%)$ adhiere a la religión católica.

162 de los encuestados dicen que les interesa la política $(61,54 \%, 95 \%$ IC: $56 \%-67,1 \%)$. Del total de participantes, un $43,48 \%$ refiere no tener las mismas ideas políticas que sus padres. Los detalles acerca de la inclinación política de los estudiantes de medicina que participaron en el estudio se muestran en la Figura 4. Sólo 16 personas $(5,35 \%)$ militan en algún partido político. Un $42,14 \%$ de la muestra (126 estudiantes, 95\% IC: $35,5 \%$ - 48,8\%) no está inscrito en el registro electoral, y de las 167 personas que sí están inscritas, la mayoría (94\%) volvería a hacerlo si tuviera la oportunidad. De los no inscritos, la mayoría $(21,43 \%)$ no lo ha hecho porque no le interesa la política.

En cuanto a la variable de confusión (desempeño de trabajos remunerados), 56 estudiantes (18,73\%, 95\% IC: $13,5 \%-24 \%)$ trabajan. De ellos, 26 alumnos $(35,71 \%)$ lo hacen en forma esporádica, siendo un porcentaje menor los que laboran más de 12 horas a la semana $(23,21 \%)$. Un $62,5 \%$ lo hace sólo para contar con un dinero extra, y el porcentaje restante lo hace para pagar sus estudios, para ayudar a su familia, o por otros motivos. Los detalles de las áreas en las que trabajan los estudiantes de medicina se muestran en la Figura 5. Un 30,96\% de los estudiantes que no trabaja, no lo hace porque no lo necesita. Un $56,07 \%$ declara no hacerlo porque no tiene tiempo. El $44,48 \%$ del total de la muestra considera que los estudiantes de medicina de primero a quinto año de la carrera tendrían suficiente tiempo para trabajar; sin embargo, sólo un 16,05\% piensa que un interno de $6^{\circ}$ ó $7^{\circ}$ año podría hacerlo.
Tabla 1. Características de los encuestados

\begin{tabular}{|c|l|}
\hline Total de encuestados & $299(100 \%)$ \\
\hline U. de Chile & $61(20,4 \%)$ \\
\hline U. Mayor & $61(20,4 \%)$ \\
\hline U. de Santiago & $61(20,4 \%)$ \\
\hline U. Austral & $53(17,73 \%)$ \\
\hline U. de Concepción & $63(21,07 \%)$ \\
\hline Edad (años) & $23,4(\mathrm{DS}: 2,85)$ \\
\hline Hombres (\%) & 52,17 \\
\hline Colegio de Egreso (\%) & \\
\hline Estatal & 13,7 \\
\hline Privado Subvencionado & 28,8 \\
\hline Privado & 56,9 \\
\hline
\end{tabular}

* El valor entre paréntesis corresponde a la desviación estándar a menos que se especifique el símbolo “\%”, que se refiere a porcentaje.

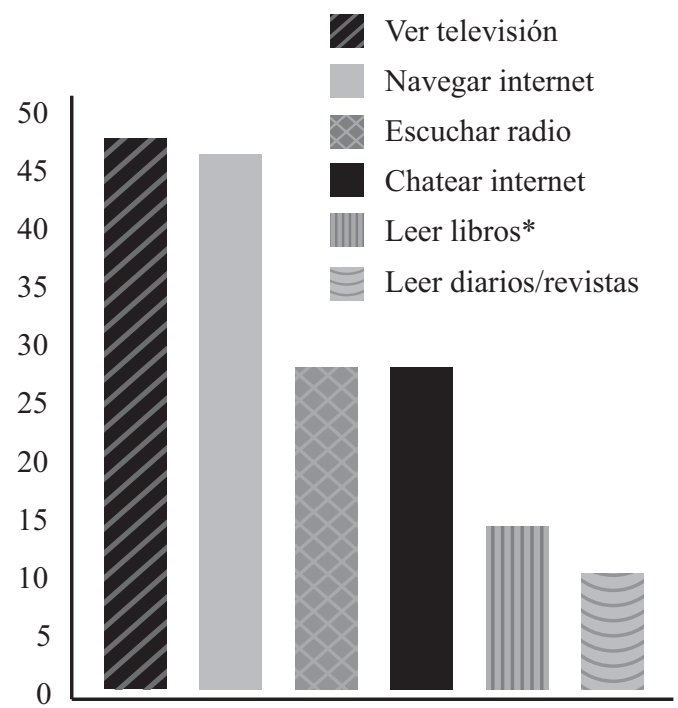

Figura 2. Porcentaje de estudiantes que dedican más de 1 hora al día a ver televisión, navegar en Internet, escuchar radio, chatear por Internet, leer libros, y leer diarios y/o revistas.

\footnotetext{
* Lectura de libros no relacionados con la carrera de Medicina.
} 

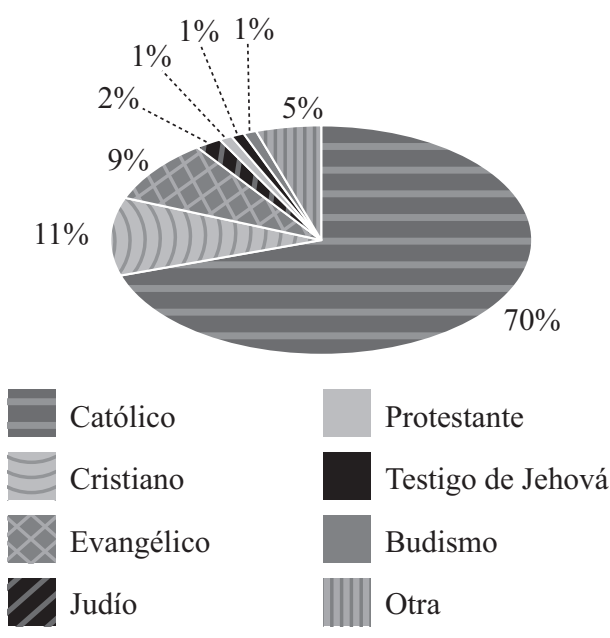

Figura 3. Religiones a las que adhieren 214 estudiantes de medicina.

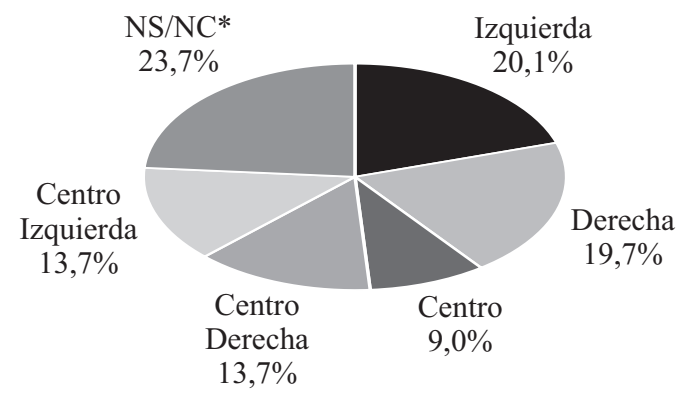

Figura 4: Inclinación política de los estudiantes de medicina.

* NS/NC: No sabe / No contesta.

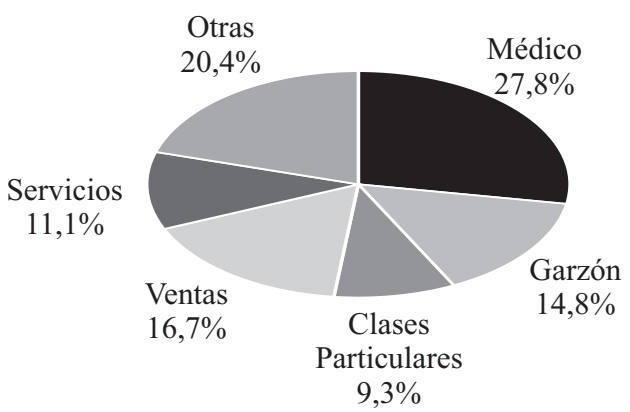

Figura 5: Trabajos remunerados: áreas en las que se desempeñan los estudiantes de medicina que trabajan.

\section{DISCUSION}

El consumo de tabaco entre los estudiantes de medicina es menor que en la población general de entre 19 a 25 años de edad (un 57,58\% de los jóvenes chilenos de ese grupo etario fuman ). La carrera produciría un factor estresor a lo largo de los años, que provocaría que los estudiantes que fuman aumenten la frecuencia de este hábito durante la carrera. El consumo de alcohol es mayor que en la población general de 19 a 25 años (un 66,13\% de la población chilena de 19 a 25 años declara consumir alcohol), pero la mayoría declara hacerlo sólo los fines de semana.

Cerca de la mitad de los estudiantes de medicina dedica parte de su tiempo libre a actividades culturales, siendo mucho más frecuente que vayan al cine que a otro tipo de manifestaciones artísticas. Menos de un quinto lee un libro al mes que no se relacione con sus estudios, lo que demuestra un bajo interés por la lectura y, probablemente, la escasez de tiempo. Las escuelas de medicina en Chile, en general, no incentivan en sus estudiantes el desarrollo de las artes y letras, por lo que estos resultados demuestran la gran falencia que existe en este aspecto.

Sólo cerca de la mitad de los encuestados realiza deportes más de una vez al mes. Esto podría explicarse, nuevamente, por una carga académica excesiva que impide la práctica de estilos de vida saludables.

Los futuros médicos presentan inquietud por informarse en actualidad nacional y cultural preferentemente, pero la gran mayoría sólo lee los periódicos los fines de semana, y cerca de un cuarto de ellos no los lee nunca. La mayoría manifiesta interés por la política, con una tendencia mayoritaria hacia el centro -centro izquierda- centro derecha, sin embargo pocos están inscritos en el Servicio Electoral (aunque el porcentaje es menor que en la población chilena de entre 19 a 24 años $^{9}$ ); y una pequeña proporción declara militar en un partido político. Debiera estudiarse la posibilidad de dar un espacio a estos jóvenes para incentivar que se informen y discutan acerca del acontecer 
nacional, internacional, y cultural a través de debates, seminarios, reuniones, y realicen cursos de humanidades que sean integrados a la malla curricular

Si bien la mayoría considera importante para la formación médica la práctica de actividades comunitarias, son pocos los que la realizan, y de ellos la mayoría ya lo hacía antes de comenzar la carrera. Existe poco interés de los estudiantes en este aspecto, y probablemente también influye la falta de tiempo y la alta exigencia académica.

El factor económico no contribuiría en estos resultados, ya que un bajo porcentaje declara hacer o tener trabajos remunerados (a pesar que muchos piensan que los estudiantes de medicina sí podrían hacerlo durante la carrera), y la mayoría de los que lo hace no es por necesidades económicas.

Actualmente las mallas curriculares de las escuelas de medicina que fueron incluidas en este estudio incorporan algunos cursos relacionados con las humanidades médicas*. La mayoría ofrece clases de antropología médica, historia de la medicina, e introducción a la medicina en los primeros años de la carrera; y otras entregan cursos electivos en estas materias. Cabe destacar que la Universidad de Chile imparte un curso de Fundamentos Antropológicos, Humanísticos y Éticos de la Medicina, que se prolonga durante los 5 primeros años de estudio, y se exige para obtener el grado de Licenciado en Medicina. Sería recomendable analizar la necesidad de impartir cursos de Humanidades Médicas en todas las escuelas de medicina del país, y evaluar el impacto que tendrían en la formación integral de los futuros profesionales.

\section{REFERENCIAS}

1. CALMAN, KENNETH C. The arts and humanities in health and medicine. Public Health 2005;119:958959.

2. GRANT, V. J. Making Room for Medical Humanities. J Med Ethics 2002;28:45-48

3. ELWYN, G; ELWYN, R. Stories We Hear and Stories We Tell: Analysing Talking Clinical Practice. BMJ 1999;318:186-188.

4. ACUÑA, L. Don't Cry for Us Argentinians: Two Decades of Teaching Medical Humanities. J Med Ethics: Medical Humanities 2000;26:66-70

5. ACUÑA, L. Teaching Humanities at the National University of La Plata, Argentina. Acad Med. 2003;78:1024-1027

6. GALANKIS, E. Letter: Medical humanities, kalology and philokalia. J of Med Ethics 2001;27:106

7. ZARRINPAR, A. Roles for Medical Students in the Political Process. MS JAMA: Editor's Note 2003; Vol $290 \mathrm{~N}^{\circ} 13$.

8. URRUTIA, L. Cuadernillo Temático $\mathrm{N}^{\circ} 2$ : Sociabilidad y Cultura Juvenil. INJUV, enero 1999.

9. INSTITUTO NACIONAL DE LA JUVENTUD (INJUV). Chile. La Eventualidad de la Inclusión: Jóvenes chilenos a comienzos del nuevo siglo. Principales Resultados Tercera Encuesta Nacional de Juventud, 2000.

10. MINISTERIO DEL INTERIOR, Consejo Nacional para el Control de Estupefacientes (CONACE), Área de Evaluación y Estudios. (2005). El Consumo de Cigarrillos en Chile, Estudios Nacionales de Drogas en Población Escolar de Chile 1995 - 2003. Mayo de 2005.

11. MINISTERIO DEL INTERIOR. Consejo Nacional para el Control de Estupefacientes (CONACE), Área de Evaluación y Estudios. (2004). Sexto Estudio Nacional de Drogas en Población General de Chile, 2004. Consumo de Drogas en Jóvenes.

Recepción : 18 nov. 2006

Aprobación : 18 dic. 2006

\footnotetext{
* Las mallas curriculares de estas Escuelas de Medicina fueron obtenidas de la página de Internet de las respectivas universidades, en junio de 2006.
}

Usted puede comentar éste y otros artículos publicados en la Revista Chilena de Salud Pública, enviando un correo electrónico a revistasp@med.uchile.cl 